\title{
Associative Remote Viewing for Profit: Evaluating the Importance of the Judge and the Investment Instrument
}

\author{
JOHN G. KRUTH \\ Rhine Research Center, Durham, North Carolina, USA \\ john.kruth@rhine.org
}

Submitted December 10, 2018; Accepted September 29, 2020; Published March 15, 2021

https://doi.org/10.31275/20211395

Creative Commons License C-BY-NC

Abstract-This study examined the importance of the judge and the particular investment selection in the associative remote viewing (ARV) process. In Protocol 1, ARV was used to predict investments and to generate funds. Three viewers made weekly predictions on Sunday about an image they would be shown on Friday. Two images were selected to represent different states of a stock (value increase or value decrease), and a judge reviewed the images and the viewers' information. Based on the judge's evaluation, a coordinator informed an investor whether to invest for the stock to rise or fall during the weekly session. Though the sessions lost funds due to a complication in the investment process, this was not the focus of the study. A second judge (Protocol 2) and a mock investment instrument (Protocol 3) were included, blinded to all study participants. The second judge ( 2 2) performed at a significantly less accurate level than the first judge $(p<.05)$, and $\mathrm{J} 2$ also performed significantly lower than could be expected by chance $(p=.02$; effect size $=-1.498$; power $>.80$ ). Both judges performed significantly differently on the target investment than on a control investment. Although this is a pilot study with a small sample size and a limited number of sessions, conclusions are that the selection of a judge, even a very experienced judge, can have a significant effect on the success of an ARV project and that judges' decisions are more affected by the target investments than by a comparable control investment. Future ARV projects are advised to qualify judges for accuracy just as they qualify viewers for accuracy.

Keywords: associative remote viewing; ARV 


\section{INTRODUCTION}

Upton Sinclair, the famous American author, watched his wife practice a form of telepathy over a period of three years. In his 1930 book, Mental Radio, Sinclair provides details of informal experiments he did where he and his wife would try to draw the same pictures when they couldn't see or communicate with each other. His wife's drawings included descriptive words or impressions of the information she was receiving. Sometimes, he would produce a number of images, seal them in paper, and randomly select one for his wife to reproduce. Though neither of them knew which drawing was selected, she was able to draw very similar images and sometimes provided accurate descriptions of the target drawing that was selected. Their early successes led them to try this with some of their friends, and they continued to have success, even at distances up to 30 miles.

Though Sinclair and his wife considered this a type of mind-tomind communication or telepathy-later popularized as a form of extra-sensory perception (Rhine, 1934) - the practice of trying to draw information received through extrasensory means may have been the beginning of the practice of remote viewing. Warcollier (1948) explored the possibility of telepathically communicating images in his book Mind to Mind, but in contrast to the Sinclairs' informal sessions, Warcollier documented formal experiments that provided a foundation for many future studies. His work implicitly introduced the concepts of a model of mind, information transfer, and signal-to-noise ratio, and his methods may have been the foundation for later studies by the U.S. Government (Swann, 2001).

In 1972 the idea of remote perception of images was revived at the Stanford Research Institute (SRI) with Hal Puthoff and Russell Targ (Puthoff \& Targ, 1976). Their work with viewers Ingo Swann and Pat Price caught the attention of the U.S. Government intelligence agencies and started a 20-year research program that is now commonly known as Project Star Gate. Research continued at SRI while a training program was developed for soldiers and intelligence officers to refine these techniques at Fort Meade, Maryland, USA (May \& Marwaha, 2018).

The program at Fort Meade produced a number of actionable intelligence sessions including information that helped to locate a Soviet 
aircraft that had gone down under a heavy jungle canopy in 1979. This information was later verified by former president Jimmy Carter in a 1995 speech (Reuters, 1995). Other information gathered by the Fort Meade viewers helped to locate hostages, and provided information valuable for new weapons systems, troop deployment, nuclear weapons testing, and anti-terrorist activities (Smith \& Moddel, 2015).

\section{Associative Remote Viewing}

"Associative Remote Viewing (ARV) is not an RV method, but rather a mode of employing RV to predict the outcome of future events with limited outcome sets" (Smith \& Moddel, 2015, p. 381).

ARV was developed to provide a practical way to examine events or activities that might be difficult to view directly or that might activate the logical processes that could repress the recognition of the psi information or result in analytical overlay. Analytical overlay (AOL) is a factor that is often recognized by remote viewers where their rational mind attempts to make sense of the information that is being received to construct a coherent image or perception. This rational process complicates the remote viewing procedure because the information being received by the viewer is being modified by a rational process rather than being perceived directly. In essence, AOL includes any rational but irrelevant activities that divert a viewer from the remote viewing task (Tart, 1979).

Often ARV projects have been used for investment purposes or to predict the outcome of a sporting event (e.g., Harary \& Targ, 1985; Rosenblatt, 2000; Smith, Laham, \& Moddel, 2014), but it can be applied in many different circumstances. In a typical ARV project, there are a limited number of possible outcomes, and one viewing target is selected to represent each outcome. The viewer attempts to view a target that they will be shown in the future, and based on the target they describe decisions that can be made about the associated activity or event.

In 1982, Harary and Targ conducted a well-publicized ARV project designed to predict changes in the silver futures commodities market. In an attempt to raise funds for future research projects, the researchers worked with an investor to generate income from investments using a modified RV protocol based on a design provided by Edwin 
May. After raising more than $\$ 100,000$ in 9 weeks using Harary as the viewer, they suspended the sessions and resumed a few months later using a slightly different protocol that prevented adequate feedback for the viewer. After just a few unsuccessful sessions, the second series was terminated (Harary, 1992).

Despite the inadequate results of the second series, this protocol has become iconic and a model for additional ARV sessions (e.g., Puthoff, 1984, pp. 121-122; Targ et al., 1995; Rosenblatt, 2000; Smith, Laham, \& Moddel, 2014). The sessions use the following basic methodology.

Early in each week, the viewer is presented with a task to describe an object that will be shown to them on Friday. The viewer completes the viewing and provides it to the researcher. The investor is asked to select two targets, one to represent the price of silver futures to rise, and a second to represent the price of silver futures to go down. The description of the target provided by the viewer is compared with the actual targets and a judge determines which target more closely resembles the viewer's description. If the selected target represents the commodity going up, an investor invests in the value rising. If the target represents the commodity going down, the investor makes the appropriate investment. The investments are made on Monday in anticipation of the value of the commodity on Friday. On Friday, the investor evaluates the actual value of the commodity, and the viewer is shown the target that represents the actual state of the investment (up or down) (Harary \& Targ, 1985).

In 2012, Kolodziejzyk published the results of a 13-year ARV investment study that he had conducted from 1998 to 2011 (Kolodziejzyk, 2012). Using a unique, computer-based approach enabled him to act as the viewer, judge, and investor for 5,677 trials. He correctly predicted his investments $52.65 \%$ of the time, which is a significant variance from chance $(z=4.0)$. Though the protocol was complicated because he combined his knowledge of the stock market with the ARV protocols he employed, the combination yielded a profit of more than $\$ 146,000$ during this time period.

Using a process similar to the Harary and Targ silver futures investments, Smith, Laham, and Moddel (2014) trained inexperienced viewers to predict whether the value of the Dow Jones Industrial 
Average would rise or fall on the day after the prediction. Seven out of seven predictions made using this protocol were correct and produced highly significant results $(p<.01)$, earning a profit of $\$ 16,000$ for two investors.

A meta-analysis by Bierman and Rabeyron (2013) combined the results of 17 ARV projects that they were able to verify as sufficiently designed and well-reported. In an evaluation of more than 550 trials, they identified a success rate of $63 \%$. In a followup study of a casino-style ARV experiment, they found that they obtained a $56 \%$ success rate, attributing their lower success rate to their automated, machine-scoring technique rather than utilizing human scorers.

Recent ARV endeavors have explored ways to modify the protocols by integrating a larger number of viewers or using computerized, session-management tools to facilitate working with a large group. Katz, Grgic, and Fendley (2018) reported on a 14-month project that involved more than 60 viewers and 177 predictions. The predictions were focused on investments in the Foreign Exchange Currency Market (FOREX). The group sessions lost nearly all of their seed capital, but a contributing factor in this may have been their inexperience with FOREX investing and the selection of their investment instrument. Despite the significant losses in this project, the study examined the performance of different teams in an attempt to develop a model for best practices when using a large group for ARV sessions. The results indicated that the teams that kept their protocols simple produced better results, and also the addition of a large number of sessions over a short time frame added stress on the traders which may have negatively affected the investment outcome.

\section{Prospective and Retrospective Studies}

Studies such as those by Bierman and Rabeyron (2013) and Katz, Grgic, and Fendley (2018) implement a retrospective analysis of data gathered during an ARV session. Bierman and Rabeyron discussed the possibility that the differences in their judging process may have accounted for differing scores. Katz, Grgic, and Fendley indicated that their choice of an investment instrument (FOREX) could have affected the outcome of their investments due to the complexity of the instrument. Other retrospective analyses (e.g., Katz, Beem, \& Bulgatz, 2014; 
Grgic, Katz, \&Tressoldi, 2018) have been presented related to ARV projects in an attempt to better understand the factors that can affect the success of a project. Retrospective studies are valuable to guide future research and identify factors that may be important to our understanding of ARV and other topics.

Prospective studies define all of the factors, variables, and analyses before the study begins. This approach increases the credibility of the study results and allows for the investigation of specific factors while limiting the influence of unanticipated activities. When a study is completely designed before it is conducted, there are fewer opportunities for bias, unconscious or conscious, to affect the analysis or interpretation of the results. Finally, prospective studies can be preregistered with a study registration database, like the Koestler Parapsychology Unit Study Registry, providing additional credibility to the scientific methodology and the resulting analysis.

Note: This study was not preregistered, but it was completely designed, specified, and reviewed before any data were collected.

\section{Purpose and Study Design}

The purpose of this study is to determine the impact of the judges' analyses and the choice of investment instrument on the success of an ARV series. Although all of the study participants recognized that the intent of the sessions was to generate revenue through investments, the scientific investigation was conducted using multiple judges and multiple investment instruments in order to analyze the results based on an evaluation of these factors rather than on the accumulation of wealth.

Many recent RV studies are focused on evaluating the performance of the viewers and exploring the factors that contribute to increasing their accuracy. This study does not examine these factors, and therefore will not report on any of the specific sessions provided by viewers or explore the success rates of the viewers. Those questions are left to other researchers who are more experienced with training methodologies and the environment that best supports viewers.

This is a prospective study that utilizes multiple judges and multiple investment instruments in order to determine if there is a difference between the results obtained when using different judges or different investing instruments. 


\section{Hypotheses}

$\mathrm{H}_{1}$ : In an ARV study, the judge's evaluation can have an impact on the accuracy of the predictions and affect the success of the investment process.

$\mathrm{H}_{2}$ : In an ARV study, the predictions made will apply to the target investment instrument more than they will apply to a randomly selected investment instrument.

\section{Design}

This study involved three concurrent activities designed to explore the hypotheses. A single data collection protocol was used to gather information, but two additional protocols were implemented to test the main hypotheses. Details are provided in the methodology section below.

\section{Protocol 1}

The preliminary data collection protocol implemented traditional ARV sessions similar to the original silver futures study completed by Harary and Targ. There is one noted modification to the original protocol in that three viewers were used instead of one, and the judge reviewed information from all three viewers to determine which target was the best match for the data gathered from the viewers. After the viewers' data was gathered, it was sent to the judge for analysis. Finally, a single investment instrument was selected for the study, and investments were made based on the impressions of the viewers and the analyses of the judges throughout the study.

\section{Protocol 2}

When data were sent to the judge, an additional protocol was added, unknown to everyone except the primary investigator (PI). Two judges were included in the study though both judges believed they were the only judge involved. The PI took the information sent to the first judge and sent it to a second judge. The results of the judging sessions were compared to determine if the evaluations of the judges could affect the results of the ARV sessions. (See note about a potential experimenter effect in the Methodology section under Protocol 2.) This protocol was designed to investigate $\mathrm{H}_{1}$. 


\section{Protocol 3}

Unknown to all participants except the PI, a second instrument was selected for comparison purposes. No investments were made for this second instrument, but the activity of this instrument was tracked throughout the study. This protocol was designed to investigate $\mathrm{H}_{2}$.

Since the analysis of this study does not include an evaluation of the money lost or gained during the sessions, the values of the instruments are reported, but are not significant in evaluating the hypotheses. The hypotheses are evaluated strictly on a comparison of the judgments made by the two judges and the difference between the predicted results and the actual results of the two investment instruments.

\section{Time Frame and Investments}

The study included 13 viewing sessions, one week for each session, over a 15-week period. The first session was a test session to verify that the communication process would work correctly and to ensure that all participants understood their roles. There were 12 weeks of experimental sessions when there were plans to make investments (see Appendix).

The investment and the mock investment were selected before the study began, and the same instruments were used throughout the study to allow for the evaluation of the effects that the instrument selection had on the success of the process $\left(\mathrm{H}_{2}\right)$. The original decision was to invest in commodities due to their high volatility over a short period of time. Since each session ran from Monday through Friday, it was important that each instrument have sufficient variability during the session to identify the effectiveness of the process and so that the change in value would give the study the best chance of producing profit during the week.

Due to investment limitations presented by the investment company selected for this study, commodity investment was not available. After investigations with the investment company, the instruments chosen were stocks with high volatility. By querying a site listing the 50 stocks with the highest volatility, two stocks with high volatility were chosen. The selection process investigated the extent of volatil- 
ity within a 5-day period, the availability of the stock, and information about whether the stock was available to be shorted - a term used for investing in a stock when you believe its value will fall during a week. The exact same process was used for one stock that would be used for investments, and a second, mock stock that would just be monitored throughout the study for comparison purposes.

The selected stocks were used throughout the study regardless of whether they rose or fell or whether the investments were successful. The consistency of the investment instruments (i.e. using the same stocks throughout the study) was essential to evaluate the factors being examined for $\mathrm{H}_{2}$.

\section{METHODOLOGY}

All participants in this study were focused on the task of generating profit from the investments that were made during the 12 weeks of the study. There were five categories of participants included in this study.

Coordinator: The coordinator performed the project tasking, collected data, and passed information among the other members of the team. The coordinator tasked the viewers, collected the viewing data, requested the targets, passed the information to the judge, determined how the investment should be placed, informed the investor, and provided feedback to the viewers once the actual target was identified.

Viewer: There were three viewers. Each viewer completed the viewing with which they were tasked, one viewing per week. They would provide their viewing information to the coordinator via email when it was completed. None of the viewers knew any of the others, and they knew the identity of the coordinator only. The viewers were tasked on Saturday and returned their information to the coordinator on Sunday evening.

Target Selector: The target selector chose the two targets that would be used to represent changes in an investment for the week. In this study, the targets were selected randomly using the ARV Studio software which had a target pool of more than 1,000 targets and a random selection process (http://arv-studio.com/). The targets were electronic images specifically selected for ARV projects to be sufficiently different to facilitate the judging process. 
Judge: The judge was provided with the viewers' information and the target images. The judge used a process that was familiar and comfortable. There were no limitations or instructions given to the judge, as they were highly experienced as a judge for ARV projects. The second judge (J2) included in this project to evaluate $\mathrm{H}_{2}$ was also highly experienced and received exactly the same instructions and information as the first judge (J1).

Investor: The investor received direction from the coordinator to invest as if the instrument was going up or down during the week. The investor would make the investment on Monday and resolve it on Friday. When the investor resolved the investment on Friday, the coordinator would be informed of the actual status of the investment at that time (up or down from the original value of the investment).

The viewers and judges in this study were very experienced in their roles and had demonstrated success in similar projects in the past. Only the coordinator and the investor had not been involved in a fully structured ARV project before, and the roles of these team members were carefully designed and structured to minimize their involvement in the portions of the process that directly involved psi processes (i.e. viewing and judging). The viewers used viewing methods that were most comfortable for them, and the judges followed a judging method that was familiar and comfortable.

\section{Session Overview: Protocol 1}

Each session began on a Saturday and was completed the following Friday (Figure 1). Investments for each week were made on Monday at 3 p.m. EST and resolved on Friday at I p.m. EST (if an investment was made for that week). The tasks included:

- The viewers were tasked on Saturday and completed viewing by Sunday evening when their session data were scheduled to be sent to the project coordinator.

The timing varied slightly for each viewer depending on the normal process used by the viewer. The viewers were not required to use a specific protocol or follow any standard procedure. They were encouraged to use a method for viewing that was familiar to them and that they felt would be most 
likely to produce an accurate viewing. Each of the three viewers in this study chose to use a different approach to viewing. After the viewing data were returned to the coordinator, the coordinator requested that the target selector (T.S.) select two target images for the week. The targets were selected after the viewing had been completed which implies a precognitive viewing process. Since the viewers were told to describe a target that would be seen on the following Friday, the viewing session was precognitive by definition, and the timing of the target selection was designed to facilitate maximum blinding rather than any consideration of when the viewers were targeted.

The two images were selected using ARV Studio software (http://arv-studio.com/) which is designed to select a pair of random targets specifically designed for use in ARV sessions. The T.S. sent the electronic images to the coordinator via email.

- The coordinator sent the targets and the viewers' descriptions/ drawings to the judge for evaluation. After they were sent, the coordinator randomly assigned investment states to the two targets using a truly random process (implemented by random.org). Zero represented the investment going down during the week, and one represented the investment going up or staying the same during the week. The process for assigning meaning to the targets was designed to maintain maximum blinding for the participants.

- Judging of the viewing information and targets was completed before Monday at $3 \mathrm{p} . \mathrm{m}$. and the results of the judging were sent to the Coordinator. The judge told the coordinator which target image was the best match for the viewers' information. If the judge determined that the viewers' data conflicted or did not match either image, the judgewould tell the coordinatorthere was no choice fortheweek(NC).

- Based on the associations that had been determined earlier in the session, the coordinator contacted the investor on Monday and indicated whether the investor should make an investment for the stock to go up or to go down, or should not make an investment for the week in the case where the judge indicated no choice (NC).

- The investor made the appropriate investment on Monday at 3 p.m. and resolved the investment on Friday at 1 p.m. 


\section{Standard ARV Protocol: Protocol 1}

\begin{tabular}{|c|c|c|c|}
\hline Saturday & Sunday & Monday & Friday \\
\hline & $\begin{array}{l}\text { Viewers send } \\
\text { impressions (text } \\
\text { descriptions/drawings) } \\
\text { to the coordinator }\end{array}$ & $\begin{array}{l}\text { Judge returns } \\
\text { evaluation of data } \\
\text { to coordinator: } \\
\text { targetA or targetB }\end{array}$ & $\begin{array}{l}\text { Investor resolves } \\
\text { investment \& } \\
\text { tells coordinator }\end{array}$ \\
\hline \multirow[t]{2}{*}{$\begin{array}{l}\text { Viewers are } \\
\text { tasked: Describe } \\
\text { image you will } \\
\text { see on Friday }\end{array}$} & $\begin{array}{l}\text { Coordinator gets } \\
\text { random target images } \\
\text { and sends targets and } \\
\text { viewers' data to judge }\end{array}$ & $\begin{array}{l}\text { Coordinator } \\
\text { determines } \\
\text { association \& } \\
\text { contacts investor }\end{array}$ & (up/down) \\
\hline & $\begin{array}{l}\text { Coordinator randomly } \\
\text { selects an association } \\
\text { for each target (rise in } \\
\text { value or fall in value) }\end{array}$ & $\begin{array}{l}\text { Investor makes } \\
\text { investment based } \\
\text { on direction of } \\
\text { coordinator }\end{array}$ & $\begin{array}{l}\text { actual } \\
\text { investment value } \\
\text { to viewers as } \\
\text { feedback }\end{array}$ \\
\hline
\end{tabular}

Figure 1. Standard ARV protocol.

- When resolving the investment on Friday, the investor would determine the actual status of the stock at that time and communicate the actual state to the coordinator. The three appropriate states were stock up, stock down, and no change.

- The coordinator would determine which image represented the actual state of the stock value, and that image would be provided to all of the viewers on Friday as feedback for the session that week.

The viewers and the judges were never informed of the investment instrument or the state of the investment each week. They also did not know whether or not the investment process was making money-i.e. the viewers and judges did not know whether their decisions resulted in gains or losses for the investments in the study. They remained blind to the entire process except for their specific tasks. The judges were never shown the feedback or told which targets were being used for investment purposes.

\section{Results of the Investment Process}

Nearly every recent ARV study that includes investments or betting evaluates the success of the project based on whether the project made a profit. When that is the primary goal of the project, this is a reasonable evaluation method. It is also a practical way to provide 
an example of a viable application for ARV and an effective means of drawing attention to the value of psi research. In evidential terms, this evaluation method is flawed. While the overall goals of a study may be met, when the results are evaluated based on the overall earnings, the details of the individual trials may be minimized or dismissed.

For example, stock investments provide an uneven earning capacity from one session to another, and a study that successfully earns money investing in stocks could earn the majority of the funds in a single trial while losing money in every other trial. Using traditional statistical evaluations, significance would be evaluated based on the number of successful trials versus the number of unsuccessful trials, but in many ARV studies the results of individual trials are minimized when the study successfully earns a profit.

This study presented an intention to earn funds, but it was not related to the evidential goals of the study. In this study, the results of individual trials were used to evaluate the performance of the judges in the study and the impact of the choice of the investment instrument. The profits or losses from the investments are provided for informational purposes, but are not considered in the evaluation of $\mathrm{H}_{1}$ or $\mathrm{H}_{2}$.

Although the investment company used for this study indicated that the stock that was selected could be shorted (i.e. an investment could be made when the stock was predicted to fall), the stock was not available to be shorted with this company. This complication prevented investments for weeks when the stock was predicted to fall (Table 1). Because of this complication, the overall value of the investment fell approximately $7.5 \%$ during the study. If the stock could have been shorted at the appropriate time, the value of the investment would have risen $2.5 \%$ instead of falling.

\section{Additional Explorations and Protocols}

Many informal discussions about remote viewing protocols discuss the importance of the judge in the process, often indicating that the judge is more important than the viewers. Also, there are some discussions around ARV that emphasize the importance of selecting the correct investment target. This additional protocol is designed to evaluate these two claims about an ARV study to measure the impact of a judge $\left(\mathrm{H}_{1}\right)$ and the selection of a target investment $\left(\mathrm{H}_{2}\right)$. 
TABLE 1

Down Weeks (Investments Could Not Be Made)

Weeks when investments were lower in value and an investment normally would have been placed. Due to complications in the investment process, no investments could be made during these weeks.

\begin{tabular}{cccccc}
\hline \hline $\begin{array}{c}\text { Week (hit/ } \\
\text { miss) }\end{array}$ & Start & End & Difference & Percent & Value \\
\hline (miss) & 11.68 & 11.28 & .40 & 3.42 & -684 \\
5 (miss) & 11.10 & 10.99 & .11 & 0.99 & -198 \\
8 (hit) & 11.26 & 11.20 & .06 & .53 & 106 \\
9 (hit) & 11.20 & 10.80 & .40 & 3.57 & 714 \\
10 (hit) & 10.87 & 10.86 & .01 & .001 & 2 \\
11 (hit) & 10.54 & 10.25 & .29 & 2.75 & 550 \\
\hline
\end{tabular}

\section{Additional Participants and Methodology: Protocol 2}

Protocol 2 was designed to evaluate $\mathrm{H}_{1}$, and it involved one additional judge $\left(\mathrm{J}_{2}\right)$ and an additional coordinator $\left(\mathrm{C}_{2}\right)$ - the primary researcher.

The original protocol was as follows:

- Saturday: 3 viewers are tasked

- Sunday evening: viewers provide viewing information to coordinator

- Sunday evening: 2 targets are selected by target selector and sent to coordinator

- Sunday evening: coordinator sends all viewing data and 2 targets to the judge

- After judge is sent information, coordinator randomly assigns values to the targets (up/down) without informing the judge of these associations

- Monday morning: judge sends decision to the coordinator

- Monday 3 p.m.: investor makes investment based on coordinator recommendation

- Friday I p.m.: investment is resolved and feedback is sent to the viewers 


\section{Protocol 2: Additional Participants}

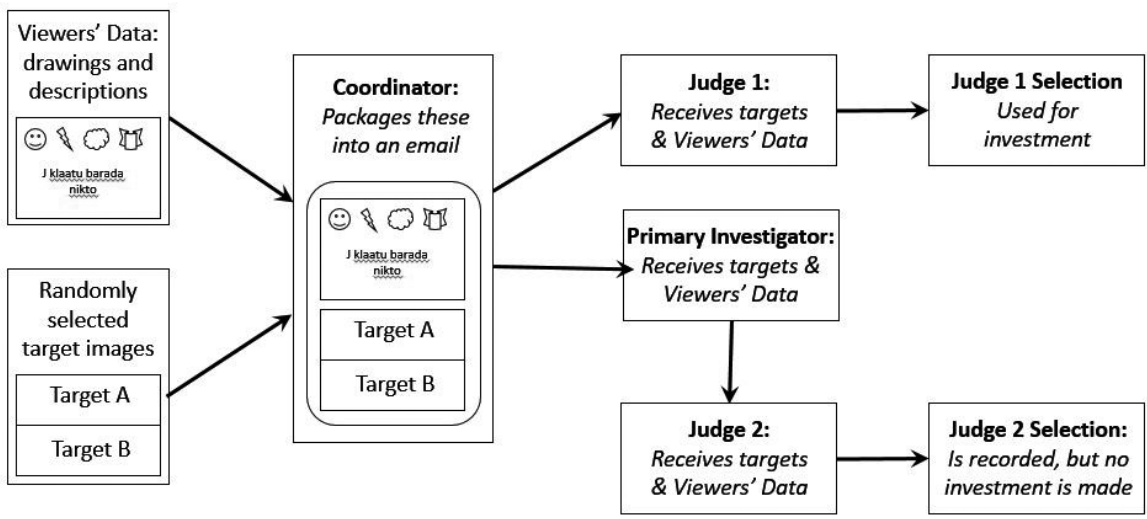

Figure 2. Protocol 2-Testing the judges.

Protocol 2 ran concurrently with Protocol 1 and for the same time frame (Figure 2). In addition to the original protocol, a second coordinator (C2) received the viewing and target information from the coordinator when it was sent to the first judge (J1). C2 took this information and sent it to a second judge ( $/ 2$ ) who determined if the viewing material matched one of the targets. J2 returned the decision to $C_{2}$ only. No investments were made based on the decisions of $\mathrm{J} 2$.

Note: Though C2 intervened as the second coordinator in the process and forwarded the information to $\mathrm{J}_{2}, \mathrm{C}_{2}$ sent exactly the same data to J2 as was sent to J1. Also, C2 had no more knowledge about the targets or data than the primary coordinator. Essentially, C2 stripped the email header from the information sent to $\mathrm{J} 1$ and sent the exact same email to J2 with no changes at all. There may have been a psi-enhanced experimenter effect introduced by this intervention, but every precaution was taken to ensure that the information sent to both judges was exactly the same and sent in exactly the same way.

Both judges involved in the study were informed that there might be an additional judge involved in the study as a backup for the primary judge, but both judges believed that they were the primary judge and that investments were being made based on their decisions. 


\section{Additional Investment Instrument: Protocol 3}

$\mathrm{C}_{2}$ also monitored a mock investment instrument where no money was involved. The mock instrument was a randomly selected stock that was different from the stock used for investment purposes. The mock instrument was selected from the same list as the original investment was and using the same methodology. The stock was selected randomly from a list of the 50 most volatile stocks and it underwent the same qualification process as the original stock. $C_{2}$ tracked the status of this stock throughout the study, but this information was not communicated to anyone involved in the study.

None of the participants in the study, including the project coordinator, knew there was a second judge or a second investment instrument. They also were not aware that there was an additional evaluation going on to determine the impact of the judge or the investment choice on ARV processes (Table 2).

TABLE 2

Judge's Performance for Each Investment Option and Total Correct Predictions for Judges and for Each Investment

The possible correct values for each judge (10 \& 8) vary because both judges indicated that no choice (NC) should be made on some of the sessions. That is, the judges determined that the viewers' information was not sufficient to make a

selection for some weeks, so no investment would be made in those weeks.

\begin{tabular}{lccc}
\hline \hline & $\begin{array}{c}\text { Investment } \\
\text { (Targeted) }\end{array}$ & $\begin{array}{c}\text { Investment } \\
\text { (Control) }\end{array}$ & $\begin{array}{c}\text { Totals for } \\
\text { Judges }\end{array}$ \\
\hline Judge 1 & $5 / 10$ correct & $6 / 10$ correct & $11 / 20$ correct \\
Judge 2 & $1 / 8$ correct & $5 / 8$ correct & $6 / 16$ correct \\
Totals for Investments & $6 / 18$ correct & $11 / 18$ correct & \\
\hline
\end{tabular}

\section{ANALYSIS}

Many remote viewing studies focus on the value and accuracy of the viewers involved in the process. Viewers are considered to be the most important component of the process and most likely to be expressing psi. This study does not dispute the importance of having 
good viewers or the value of the viewers in an Associative Remote Viewing process.

This study was designed to determine whether there was a significant difference based on the judges who were involved in the sessions $\left(\mathrm{H}_{1}\right)$. In addition, this study was designed to determine if there was a difference between performance on a targeted stock or a stock that was chosen at random and not targeted for the viewers $\left(\mathrm{H}_{2}\right)$. In the analysis that follows, J1 represents Judge 1 and J2 represents Judge 2.

Some readers may consider the ARV sessions to be unsuccessfully supported if a profit was not made from this study and question whether there is any value in further evaluation of the judges $\left(\mathrm{H}_{1}\right)$ and the investment instrument $\left(\mathrm{H}_{2}\right)$. Though there was an intention to generate funds from this study, the primary goal of the study was the evaluation of $\mathrm{H}_{1}$ and $\mathrm{H}_{2}$, and the primary goals are unaffected by the amount of profit generated. The profit generated from this study and the value of the investments are not used in the analysis of the hypotheses. For more information about why profits were not used in this analysis, see the section above on the Results of the Investment Process.

\section{Initial Analysis Design}

The initial design called for an analysis of the differences between the decisions made by J1 and J2. This analysis would be completed by doing a means comparison using an independent sample $t$-test to compare the number of correct decisions made by each judge $\left(\mathrm{H}_{1}\right)$. This comparison would be completed for the experimental stock (SE) and the control stock (SC) in order to evaluate $\mathrm{H}_{2}$.

It was anticipated that there would be some sessions where the viewers' impressions would not match the selected targets well enough for the judges to make a clear distinction about which target matched the impressions. If this happened, it could be due to the viewers producing incomplete or inaccurate impressions, or it could be due to the judges misjudging the viewers' data. In these cases, the judges were given the option of indicating no choice (NC) indicating that the judge could not make a decision based on the data provided.

There are currently no set standards for evaluating no choice (NC) decisions by the judges. Some evaluators consider a NC decision by a 
judge to be a miss, and others believe that NC should be left out of the evaluation (J. Lane, personal communication, 2018). For the purposes of this study, when the judge made an NC decision, it will be considered that this occurred because the viewers did not provide data that matched with either target, giving the benefit of the doubt to the judge. In these cases, the NC decisions will be excluded from the evaluations. Those cases will be dropped from the dataset, and only the cases where the judge made a specific decision will be included in the analysis.

\section{Summary of Initial Analysis}

The initial analysis revealed a significant difference between the judges, especially related to the targeted stock. Though J1 performed slightly higher than the expected mean across all of the sessions, the performance of $\mathrm{J} 1$ was not significantly different from chance. On the other hand, J2 performed at a level significantly lower than chance, but only on the targeted stock. Both judges performed at chance for the non-targeted or control stock (see Table 2).

\section{RESULTS \\ COMPARING JUDGES AND INVESTMENT INSTRUMENTS}

In an evaluation of $\mathrm{H}_{1}$, J1 outperformed $\mathrm{J}_{2}$ in predictions $(\mathrm{p}=.05$ one-tailed in an independent sample $t$-test). This is due to the very low prediction scores for $\mathrm{J}_{2}(\mathrm{l} / 8$ with 4 No Calls). This was only the case with

\section{TABLE 3}

\section{Judges' Performance and Comparison}

The difference between the means is just at the threshold level for significance. Significance and equality of variance calculated using an independent sample $t$-test and adjusted due to unequal variances calculated using Levene's Test for Equality of Variance.

\begin{tabular}{lccccc}
\hline \hline & Mean & N & SD & Variance & Significance \\
\hline Judge 1 & 0.50 & 10 & 0.527 & $F=11.43$ & $\begin{array}{c}p=.05 \\
\text { (one-tailed) }\end{array}$ \\
\hline Judge 2 & 0.125 & 8 & 0.354 & $p=.004$ & P \\
\hline
\end{tabular}


the Experimental Stock, SE (J1 mean $=.50, \mathrm{~N}=10, \mathrm{SD}=0.527$; $\mathrm{J} 2$ mean $=.125, \mathrm{~N}=8, \mathrm{SD}=0.354$ ) (see Table 3).

The initial evaluation of $\mathrm{H}_{2}$ indicates that the number of correct predictions for the experimental stock was slightly lower than for the control stock (SE = 6/18; SC $=11 / 18)$. The difference was just outside the significance threshold $(p=.051$ one-tailed with an independent sample $t$-test).

This initial analysis, based on the predetermined analysis methods, supported $\mathrm{H}_{1}$, indicating that there is a difference in study results based on the judge that is selected and the evaluation produced by a particular judge $(p=.05) \cdot \mathrm{H}_{2}$ was marginally unsupported or nearly supported $(p=.051)$, indicating that there may be a difference in results based on the instrument that is targeted versus a randomly selected investment instrument that is not targeted by the participants.

\section{Post Hoc Analyses}

During the study, J1 correctly predicted the state of the stock 4 weeks in a row. The probability of this is 1 in 16 or 0.0625 . J2 incorrectly predicted the state of the stock 9 weeks in a row. The probability of this is 1 in 512 or 0.002. Though not specifically related to a means comparison used for the evaluations, these streaks of correct predications by J1 and incorrect predictions by $\mathrm{J} 2$ add evidence to indicate that there is a strong difference between the judges.

In post hoc analysis, J2 demonstrated a significant tendency to make incorrect judgments which could be interpreted as psi missing or an exceptional string of bad luck. Total: $1 / 8$ correct; mean $=0.125$;

\section{TABLE 4}

\section{Evaluation of J2's Predictions for the Target Investment}

Significance level calculated using a one-sample $t$-test with an expected mean of 0.5

\begin{tabular}{lcccccc}
\hline \hline & Mean & N & SD & Significance & Effect Size & Power \\
\hline Judge 2 & 0.125 & 8 & 0.354 & $p=.02$ & $d=-1.498$ & $>.95$ \\
\hline
\end{tabular}


$p=0.02$ for one sample $t$-test with an expected mean of $0.5 ; \mathrm{D}=$ -1.498; power > .99 (see Table 4).

Additional post hoc analyses were evaluated during weeks when the stocks changed significantly, and two levels were selected: delta $>2 \%$ and delta $>1 \%$. In both cases, $\mathrm{J} 2$ continued to demonstrate a significant tendency toward incorrect judgments regarding the state of the stock at the end of the week. Delta $>2 \%: 0 / 5$ correct; mean $=0.0$; $p$ is undefined because the mean is 0 . Delta $>1 \%: 1 / 7$ correct; mean $=0.143 ; p=.047$ with a one sample $t$-test with an expected mean of 0.5 .

When examining the judges' results only during weeks when stocks made significant changes (delta $>1 \%$ or delta $>2 \%$ ), there was no significant difference in the judges' performances and no significant differences between the predictions made for the experimental versus the control stocks ( $p>.05$ in all cases).

\section{CONCLUSIONS}

This was a pilot study with a very small sample and a limited number of sessions. It should be replicated, and additional evaluations of ARV projects should be completed to confirm these findings. The methodology and analysis methods used in this study vary from many recent ARV studies that focus on the total amount of profit generated to determine if the sessions successfully met their goal. These modified design elements are provided as a guide for future prospective studies that explore the factors that may impact the results of an ARV session and the significance of those sessions.

All viewers and all judges involved in this study were very experienced and extremely well-respected for their knowledge and abilities with viewing and/or judging. Though this is a small sample size and a small number of sessions, it is clear that the choice of judges can have a significant impact on the results of an ARV study. The judging methods varied ( $J_{1}$ used an intuitive judging method which was completed quickly; J2 used a more procedure-based method based on the Targ Scale and evaluated the target selection based on significant differences between the ratings), but this study was not designed to evaluate one judging method against another. It would be incorrect to assume that one judging method is superior to another based on the results of this study. 
By randomly selecting a control investment to compare with the target investment, this study demonstrates that the targeted investment had a marginally more significant impact on the results than the control investment. When the judges' decisions were applied to the control investment, the results were nearly chance, but when they were applied to the target investment, the decisions made by $\mathrm{J} 2$ showed a very strong variation from chance although they were in the negative direction.

In summary, the decisions of the judges varied significantly with regard to the targeted investment, but were nearly at chance for the control investment. Also, when focusing on the targeted investment, J2 demonstrated a strong influence toward incorrectly predicting the market performance while J1 performed at chance. This study demonstrates that the choice of judges is extremely important when performing an ARV study and that judges appear to respond more strongly to a targeted investment than to a random investment that is not targeted.

This study appears to have identified an effect that is produced by different judges in an ARV project. Additional studies should be pursued to determine if certain judging protocols are more accurate than others and to what extent the judges play a role in the results of an ARV project. More importantly, future studies or projects using ARV should consider evaluating the performance of the judges who have been involved in previous ARV studies. It is important to be cautious in the selection of viewers for ARV projects, but the judges also can have a strong effect on the outcome of the project. Even judges who are welltrained and familiar with judging protocols may produce inaccurate results that could influence the results of the study.

\section{REFERENCES}

Bierman, D. J., \& Rabeyron, T. (2013, August 8-11). Can psi research sponsor itself? Simulations and results of an automated ARV-casino experiment [Paper presentation]. 56th Annual Convention of the Parapsychological Association, Viterbo, Italy.

Grgic, I., Katz, D. L., \& Tressoldi, P. (2018). The associative remote viewing rejudging project [Paper presentation]. 61st Annual Convention of the Parapsychological Association, Petaluma, CA, United States.

Harary, K. (1992). The goose that laid the silver eggs: A criticism of psi and silver 
futures forecasting. Journal of the American Society for Psychical Research, 86, $375-409$.

Harary, K., \& Targ, R. (1985). A new approach to forecasting commodity futures. Psi Research, 4, 79-85.

Katz, D. L., Beem, L. W., \& Bulgatz, M. (2014, June 27-29). What's new? Lessons learned in recent remote viewing research [Paper presentation]. International Remove Viewing Association Conference, Las Vegas, Nevada, United States.

Katz, D. L., Grgic, I., \& Fendley, T. W. (2018). An ethnographic assessment of Project Firefly: A yearlong endeavor to create wealth by predicting FOREX currency moves with associative remote viewing. Journal of Scientific Exploration, 32(1), 21-54.

Kolodziejzyk, G. (2012). Thirteen-year associative remote viewing results. Journal of Parapsychology, 76, 349-367.

May, E., \& Marwaha, S. B. (2018). The Star Gate archives: Reports of the United States government sponsored psi program, 1972-1995. Volume 1: Remote Viewing, 1972-1984. McFarland.

Puthoff, H. E. (1984). ARV applications. Research in parapsychology. Scarecrow Press. Puthoff, H. E., \& Targ, R. (1976). A perceptual channel for information over kilometer distances: Historical perspective and recent research. Proceedings of the IEEE, 64(3), 10.

Reuters. (1995, September 21). Psychic helped locate downed U.S. plane, ex-president says.

Rhine, J. B. (1934). Extra-sensory perception. Boston Society for Psychical Research.

Rosenblatt, M. (2000, April-June). Applications: AVM precognition project: Summary of results for protocol-1. Connections through Time, 7.

Sinclair, U. (1930). Mental radio. Charles C. Thomas.

Smith, C., Laham, D., \& Moddel, G. (2014). Stock market prediction using associative remote viewing by inexperienced remote viewers. Journal of Scientific Exploration, 28(1), 7-16.

Smith, P. H., \& Moddel, G. (2015). Applied psi. In E. Cardeña, J. Palmer, \& D. Marcusson-Clavertz (Eds., Parapsychology: A handbook for the 21st century, (pp. 380-388). McFarland.

Swann, I. (2001). Preface. In R. Warcollier, Mind to mind (pp. ix-xv). Hampton Roads.

Targ, R., Katra, J., Brown, D., \& Wiegand, W. (1995). Viewing the future: A pilot study with an error-detecting protocol. Journal of Scientific Exploration, 9(3), 367-380.

Tart, C. (1979). Improving real-time ESP by suppressing the future: Trans-temporal inhibition. In C. Tart, H. Puthoff, \& R. Targ (Eds.), Mind at large (pp. 139-174). Praeger.

Warcollier, R. (1948). Mind to mind. Hampton Roads. 


\section{APPENDIX}

\section{ARV For Profit—Data Summary}

August 7-11, 2017: Pilot Test to Adjust Protocol

Timeframe: Aug. 14-Nov. 10; skipped Sept. 4-8 due to holiday

$$
\begin{aligned}
\text { Exp } & =\text { Experimental Investment Instrument (used for investment) } \\
\mathrm{Ctrl} & =\text { Control Investment Instrument (not used for investment) } \\
\mathrm{J} 1 \mathrm{H} & =\text { Judge } 1 \text { Hit (correct prediction) } \\
\mathrm{J}_{1} \mathrm{M} & =\text { Judge } 1 \text { Miss (incorrect prediction) } \\
\mathrm{J}_{2} \mathrm{H} & =\text { Judge } 2 \mathrm{Hit} \\
\mathrm{J}_{2} \mathrm{M} & =\text { Judge } 2 \text { Miss } \\
\mathrm{NC} & =\text { No call by the judge (no investment made) }
\end{aligned}
$$

Aug. 14-18: Week 1

\section{Exp \%Chng}

Aug. 21-25: Week 2

Aug. 28-Sept 1: Week 3

Sept. 4-8: Skipped (Holiday)

Sept. 11-15: Week 4

Sept. 18-22: Week 5

Sept. 25-29: Week 6

Oct. 2-6: Week 7

Oct. 9-13: Week 8

Oct. 16-20: Week 9

Oct. 23-27: Week 10

Oct. 30-Nov 3: Week 11

Nov. 6-10: Week 12

\section{Experimental Stock 1:} Judge

1

2

CommonScores

(both judges in

one week)

Control Stock 2:

Judge

1

2

Common Scores

(both judges in one week)

$3.57 \mathrm{~J} 1 \mathrm{H} \mathrm{J} 2 \mathrm{M}$
1

2
$0.74 \mathrm{~J}_{1} \mathrm{M} \mathrm{J}_{2} \mathrm{H}$

Ctrl \%Chng

0.74 J1\& J2 NC

$1.67 \mathrm{~J} 1 \mathrm{H} \mathrm{J} 2 \mathrm{H}$

$0.79 \mathrm{~J}_{1} \mathrm{M} / 2 \mathrm{H}$

4.34 J1\& J2 NC

$2.06 \mathrm{~J}_{1} \mathrm{H} \mathrm{J} 2 \mathrm{H}$

$3.43 \mathrm{~J} 1 \mathrm{M} J 2 \mathrm{NC}$

$1.15 \mathrm{~J} 1 \mathrm{H} \mathrm{J} 2 \mathrm{NC}$

$0.99 \mathrm{~J} 1 \mathrm{M} \mathrm{J} 2 \mathrm{M}$

$2.66 \mathrm{~J}_{1} \mathrm{M} \mathrm{J} 2 \mathrm{M}$

$2.60 \mathrm{~J} 1 \& \mathrm{~J} 2 \mathrm{NC} \quad 46.87 \mathrm{~J} 1 \& \mathrm{~J} 2 \mathrm{NC}$

$0.73 \mathrm{~J}_{1} \mathrm{M} \mathrm{J}_{2} \mathrm{NC}$

$7.12 \mathrm{~J}_{1} \mathrm{M} \mathrm{J}_{2} \mathrm{NC}$

$0.53 \mathrm{~J}_{1} \mathrm{H} J 2 \mathrm{M}$

$2.35 \mathrm{~J} 1 \mathrm{M} \mathrm{J} 2 \mathrm{H}$

$3.70 \mathrm{~J} 1 \mathrm{H} \mathrm{J} 2 \mathrm{M}$

$0.00 \mathrm{~J}_{1} \mathrm{H} \mathrm{J}_{2} \mathrm{M}$

$1.41 \mathrm{~J}_{1} \mathrm{H} \mathrm{J} 2 \mathrm{M}$

$2.75 \mathrm{~J}_{1} \mathrm{H} \mathrm{J} 2 \mathrm{M}$

$5.84 \mathrm{~J}_{1} \mathrm{M} \mathrm{J}_{2} \mathrm{H}$

$3.62 \mathrm{~J} 1 \mathrm{M} \mathrm{J} 2 \mathrm{M}$

$9.41 \mathrm{lH} \mathrm{J} 2 \mathrm{H}$

Hits Misses No Call Cumulative

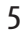

1

1

3

2

$-2$

5

7

2

4

$-6$

$\begin{array}{cccc}\text { Hits } & \text { Misses } & \text { No Call } & \text { Cumulative } \\ 6 & 4 & 2 & 2 \\ 5 & 3 & 4 & 2\end{array}$

